\title{
Efeito de dietas artificiais para a alimentação de adultos de Bonagota cranaodes (Meyrick) (Lepidoptera: Tortricidae), em laboratório
}

\author{
Effect of artificial diets for the adults of Bonagota cranaodes feeding \\ (Meyrick) (Lepidoptera: Tortricidae), in laboratory
}

\author{
Fabiana Lazzerini da Fonseca ${ }^{1}$ Silvana Manfredi -Coimbra ${ }^{2}$ \\ Josemar Foresti ${ }^{3}$ Adalecio Kovaleski ${ }^{4}$
}

\section{RESUMO}

\begin{abstract}
Adultos de Bonagota cranaodes (Meyrick) (Lepidoptera: Tortricidae) submetidos a tratamentos com água, solução de mel a $10 \%$, solução de mel a 10\% + 25\% de cerveja e sem alimento foram mantidos em gaiolas de $P V C$ transparente para avaliar a fecundidade, longevidade $e$ viabilidade de ovos em laboratório a $25 \pm 1^{\circ} \mathrm{C}$ e $70 \pm 10 \%$ de UR. Maior longevidade de fêmeas e machos e número de ovos/fêmea foram obtidos nos substratos compostos de mel a $10 \%$ (17,75 dias, 17,35 dias e 14,07 ovos/postura) e mel a $10 \%+25 \%$ de cerveja (18,25 dias, 18,20 dias e 12,71 ovos/ postura). A viabilidade dos ovos e a duração do período embrionário foram semelhantes em todos os tratamentos. $O$ substrato alimentar mel a $10 \%+25 \%$ de cerveja, apresentou efeitos semelhantes ao substrato composto de apenas mel a $10 \%$ sobre a longevidade, fertilidade e viabilidade dos ovos. Entretanto, devido ao menor custo, a dieta a base de mel apresenta melhor potencial de utilização para manutenção da criação do inseto em laboratório.
\end{abstract}

Palavras-chave: lagarta enroladeira da maçã, parâmetros biológicos, longevidade.

\section{ABSTRACT}

Adults of Bonagota cranaodes (Meyrick) (Lepidoptera: Tortricidae) were maintained in cages of transparent PVC without food, with water, and fed with honey solution at $10 \%$ and with honey solution at $10 \%+25 \%$ of beer only with water and without feeding to evaluate their fecundity, longevity and viability of eggs in growth to $\left(25 \pm 1^{\circ} \mathrm{C}\right.$ and $70 \pm 10 \%$ of $R U$ ). The longevity of females and males was longer and the number of eggs/female were obtained in the mean substratum composed of honey at $10 \%(17,75$ days, 17,35 days and 14,07 eggs/posture) and honey at $10 \%+25 \%$ of beer $(18,25$ days, 18,20 days and 12,71 eggs/posture). The viability of the eggs and the duration of the embryonic period was similar in all the treatments. The mean with honey at $10 \%+25 \%$ of beer, presented effects similar to the mean composed with honey at $10 \%$ regarding to the longevity, fertility and viability of eggs of B. cranaodes. However, due to the lower cost, the diet based only in honey represents the best choice in laboratory for maintenance rearing of this insect.

Key words: apple leafroller, biological parameters.

\section{INTRODUÇÃO}

Os seres vivos em geral são um reflexo do que consomem e, no caso dos insetos, muitos aspectos de sua biologia, incluindo o comportamento, a fisiologia e a ecologia estão, de uma ou outra maneira inserida num contexto nutricional (SCRIBER \& SLANSKY, 1981). Segundo PANIZZI \& PARRA(1991), este fato evidencia a importância do alimento para os insetos, de modo que a quantidade e a qualidade do alimento consumido na fase larval afetam a taxa de crescimento, o tempo de desenvolvimento, peso do corpo, sobrevivência, e influenciam na fecundidade, longevidade, movimentação e capacidade de

\footnotetext{
${ }^{1}$ Departamento de Zoologia, Setor de Ciências Biológicas Universidade Federal do Paraná, CP 19020, 81531-980, Curitiba, PR, Brasil. E-mail: fabifonseca12@hotmail.com. Autor para correspondência.

${ }^{2}$ Departamento de Fitossanidade, Universidade Federal de Pelotas (UFPel), Faculdade de Agronomia Eliseu Maciel (FAEM), CP 354, 96001-970, Pelotas, RS, Brasil. E-mail: silvana.coimbra@pop.com.br.

${ }^{3}$ Departamento de Fitossanidade, UFPel, FAEM, CP 354, 96001-970, Pelotas, RS, Brasil. E-mail: josemar.foresti@pionner.com.

${ }^{4}$ Embrapa Uva e Vinho, CP 1513, 95200-000, Vacaria, RS, Brasil. E-mail: adalecio@cnpuv.embrapa.br.
} 
competição de adultos, sendo que, neste caso, está principalmente relacionada à função de reprodução.

A nutrição de insetos assume importância em virtude da necessidade crescente de sua produção massal visando a resolução de problemas relacionas à entomologia básica e aplicada. Assim, o conhecimento da dieta adequada tanto à fase larval e dos adultos da espécie que se está pesquisando é fundamental para manutenção de seu nível populacional constante no laboratório.

A nutrição de adultos de insetos holometabólicos é pouco conhecida. De modo geral, para Lepidoptera, considerava-se que água e carboidrato, em concentrações variáveis, eram suficientes para a obtenção de grande quantidade de ovos em laboratório. Trabalhos mais recentes mostram que, para a obtenção de adultos mais longevos e fecundos, além da alimentação com soluções de carboidratos, devem ser adicionados à sua dieta diversos outros nutrientes, tais como sais, minerais, lipídeos, aminoácidos e vitaminas (PARRA, 2000). A respeito destes avanços, para lepidópteros em geral, considera-se que a adição de carboidratos (principalmente sacarose) em concentrações em torno de $10 \%$ é suficiente para aumentar a longevidade e obter-se o máximo potencial reprodutivo. No entanto, vários estudos foram realizados para determinar a qualidade de dietas artificiais utilizadas na criação de imaturos de Lepidoptera. EIRAS et al. (1994) e PARRA et al. (1995) testaram diferentes dietas artificiais para $a$ lagarta enroladeira da maçã Bonagota cranaodes (Meiryck) avaliando sua performance. GARCIA (1998) avaliaram a adequabilidade de três dietas artificiais para Ecdytolopha aurantiana (Lima) através da tabela de vida.

Fêmeas adultas de Lepidoptera necessitam de alimentos protéicos, sais, nutrientes, vitaminas, carboidratos, lipídeos para continuar a postura e assim obter longevidade e fecundidade ótimas (PANIZZI \& PARRA, 1991). Trabalhos mais recentes mostram que, para a longevidade e fecundidade, as fêmeas requerem sais os mais variados, lipídios e vitaminas em adição a aminoácidos ou proteínas (PARRA, 2000). Muitos adultos de Tortricidae requerem somente água para uma longevidade e fecundidade normais, embora algumas espécies necessitem açúcar para alcançar a maior produção de posturas. Em Epinotia aporema (Wals.) quando fêmeas se alimentam com $10 \%$ de solução de mel colocam 15 vezes mais ovos de quando somente alimentadas com água (BENZ, 1991). Os efeitos da presença ou não destes nutrientes na dieta de adultos de Tortricidae ainda não foram estudados.
Assim, este trabalho teve como objetivo, avaliar o efeito de diferentes substratos alimentares para adultos da lagarta enroladeira da maçã $\boldsymbol{B}$. cranoades, sobre sua longevidade, fertilidade e viabilidade de ovos.

\section{MATERIAL E MÉTODOS}

O presente trabalho foi conduzido no Laboratório de Biologia de Insetos do Departamento de Fitossanidade (DFs.), da Faculdade de Agronomia Eliseu Maciel (FAEM), na Universidade Federal de Pelotas (UFPel), à temperatura de $25 \pm 1^{\circ} \mathrm{C}$, umidade relativa de $70 \pm 10 \%$ e fotofase de $12 \mathrm{~h}$.

O experimento foi realizado a partir de adultos obtidos da criação de manutenção conduzida sobre dieta artificial desenvolvida por PARRA et al. (1995) para a lagarta enroladeira. Adultos recém emergidos foram sexados, separados em casais (um por gaiola) transferidos para gaiolas de PVC transparente, $9,5 \mathrm{~cm} \times 7,5 \mathrm{~cm}$, e submetidos a três formulações de alimento, conforme metodologia proposta por BAVARESCO (2000). Os tratamentos utilizados neste trabalho foram: sem alimento $\left(D_{1}\right)$; água $\left(\mathrm{D}_{2}\right)$; solução de mel a $10 \%\left(\mathrm{D}_{3}\right)$ e solução de mel a $10 \%+25 \%$ de cerveja $\left(\mathrm{D}_{4}\right)$. Foram avaliadas as longevidades de fêmeas, longevidade de machos, número de ovos por fêmea, viabilidade dos ovos, período de pré-oviposição, período de pós-oviposição e período embrionário.

As dietas foram fornecidas por capilaridade, em rolos dentais de algodão mergulhados em frascos plásticos, os quais foram envolvidos por cubos de isopor, de modo que ficassem fixados dentro da gaiola evitando-se dessa forma a postura nesses locais.

Diariamente, as posturas foram marcadas com caneta de transparência. A cada dois dias, foram retiradas e as dietas renovadas, para evitar alterações no comportamento do inseto e a desidratação dos ovos. As massas de ovos obtidas foram acondicionadas em vidro de $2,5 \times 8,5 \mathrm{~cm}$ e mantidas em câmara climatizada a $25 \pm 1^{\circ} \mathrm{C}$ e umidade relativa de $70 \pm 10 \%$ contendo um pedaço de papel filtro umedecido com água destilada.

O delineamento experimental empregado foi inteiramente casualizado com 20 repetições por tratamento. Cada unidade experimental foi constituída de uma gaiola com um casal e a dieta correspondente. Em função de perdas de repetições para alguns dos tratamentos, foi utilizado o cálculo da soma de quadrados para dados não balanceados através do procedimento GLM, o qual utiliza o método dos quadrados mínimos e, as médias foram comparadas 
pelo teste de Scheffé em nível de 5\% de probabilidade de erro a partir do programa estatístico SAS (SAS INSTITUTE, 1985).

\section{RESULTADOS E DISCUSSÃO}

Houve diferença significativa entre os efeitos dos tratamentos para os parâmetros longevidade de fêmeas, longevidade de machos, número de ovos/fêmea, período de pós-oviposição e período de pré-oviposição. Exceto na ausência de alimento, os efeitos dos tratamentos foram semelhantes para todos os parâmetros avaliados (Tabela 1).

As fontes nutricionais fornecidas aos adultos apresentaram influência sobre a longevidade de fêmeas e machos, onde estes quando não receberam alimentação foram menos longevos (Tabela 1). FONSECA et al., 1998 estudando a influência da dieta na sobrevivência e fecundidade de adultos da lagarta enroladeira verificaram que a longevidade máxima dos adultos foi aumentada de 11 dias (sem alimento) para cerca de 25 dias (com alimento), sendo que a fonte alimentar (água e água + mel) não interferiu no potencial reprodutivo da lagarta enroladeira. No entanto segundo PARRA et al. (1999), adultos de Diatraea saccharalis (Fabr.) alimentados apenas com água apresentaram longevidade superior àquela dos adultos alimentados com solução de carboidratos. No presente trabalho a longevidade para ambos os sexos foi maior quando os adultos foram alimentados com solução de mel a 10\% + cerveja a 25\% (D4) e com mel a 10\% (D3).

Os resultados do presente trabalho diferem dos obtidos por BAVARESCO (2000) que, estudando a exigência nutricional de adultos de Spodoptera cosmioides (Walker) (Spodoptera latifascia) com as mesmas fontes nutricionais, verificou que a longevidade de fêmeas não foi influenciada pelo alimento e que, para os machos, a longevidade foi menor quando estes não receberam alimentação.

A longevidade de machos e fêmeas seguiu a distribuição de Weibull em todos os tratamentos e a taxa de mortalidade aumentou com o tempo (Figura $1 \mathrm{e}$ 2)(SGRILLO, 1982).

A viabilidade de ovos foi superior para a dieta contendo solução de mel 10\% + 25\% de cerveja, porém não diferiu estatisticamente dos demais tratamentos. PARRA et al. (1999) encontraram para $\boldsymbol{D}$. saccharalis uma média de ovos por fêmea semelhante quando alimentada apenas com água e com várias fontes energéticas diferentes. BAVARESCO (2000), trabalhando com $S$. consmioides, encontrou uma viabilidade de $41,6 \%$ quando os adultos foram alimentados com mel a $10 \%$. Os resultados do trabalho são semelhantes ao apresentando por BAVARESCO (2000). No mesmo trabalho o autor verificou uma baixa viabilidade (2,8\%) para os adultos desprovidos de alimento.

Os períodos de pré-oviposição e pósoviposição apresentaram diferença significativa no diferentes tratamentos. O período foi menor quando os insetos não receberam alimentação (Tabela 1). O alongamento do período de oviposição, de acordo com a alimentação, já foi observada em outros lepidópteros (PARRA, 2000). O período embrionário variou de 7,0 dias para adultos sem alimento até 7,24 dias para adultos alimentados com solução de mel a $10 \%$, não apresentando diferença.

No presente trabalho, as dietas com solução de mel a $10 \%$ acrescentados de cerveja a $25 \%$ apresentaram os melhores resultados concordando com os resultados obtidos por SILVA et al. (1991) quando estudaram diferentes dietas na biologia de Mocis latipes (Guenée) concluindo que o mel é o alimento mais completo, proporcionando, em solução aquosa a 5\%, o maior período de oviposição (10 dias)

Tabela 1 - Longevidade de fêmeas (LONF), longevidade de machos (LONM), número de ovos por fêmea (NOF), viabilidade dos ovos (VO), período de pré-oviposição (PO), período de pós-oviposição (PPO) e período embrionário (PE) dos parâmetros biológicos de adultos de Bonagota cranaodes criados sem alimento $\left(\mathrm{D}_{1}\right)$, alimentados com água $\left(\mathrm{D}_{2}\right)$, solução de mel $10 \%\left(\mathrm{D}_{3}\right)$ e solução de mel $10 \%+25 \%$ de cerveja $\left(\mathrm{D}_{4}\right)$, na temperatura de $25 \pm 1^{\circ} \mathrm{C}$ e $70 \pm 10 \%$ de UR e fotofase de $12 \mathrm{~h}$, em Pelotas-RS, 2000.

\begin{tabular}{|c|c|c|c|c|c|c|c|}
\hline \multirow{2}{*}{ Tratamentos } & \multicolumn{7}{|c|}{ Parâmetros biológicos } \\
\hline & LONF & LONM & NOF & VO & PO & PPO & $\mathrm{PE}$ \\
\hline $\mathrm{D}_{1}$ & $5,80 \mathrm{c}$ & $3,85 \mathrm{c}$ & $3,03 \mathrm{~b}$ & 28,23 a & $2,28 \mathrm{~b}$ & $1,33 \mathrm{~b}$ & $7,00 \mathrm{a}$ \\
\hline $\mathrm{D}_{2}$ & $11,85 \mathrm{~b}$ & $13,65 \mathrm{~b}$ & $12,74 \mathrm{a}$ & 35,99 a & $3,55 \mathrm{ab}$ & $4,41 \mathrm{~b}$ & $7,05 \mathrm{a}$ \\
\hline $\mathrm{D}_{3}$ & $17,75 \mathrm{a}$ & $17,35 \mathrm{a}$ & $14,07 \mathrm{a}$ & 47,27 a & $2,88 a b$ & 9,71 a & $7,24 \mathrm{a}$ \\
\hline $\mathrm{D}_{4}$ & 18,25 a & 18,20 a & $12,71 \mathrm{a}$ & 33,14 a & $5,13 \mathrm{a}$ & 10,38 a & $7,20 \mathrm{a}$ \\
\hline CV (\%) & 36,23 & 30,19 & 66,75 & 52,17 & 65,76 & 55,80 & 3,82 \\
\hline
\end{tabular}

${ }^{1}$ Médias não seguidas de mesma letra na coluna diferem entre si pelo teste de Scheffé a 5\% de probabilidade de erro 

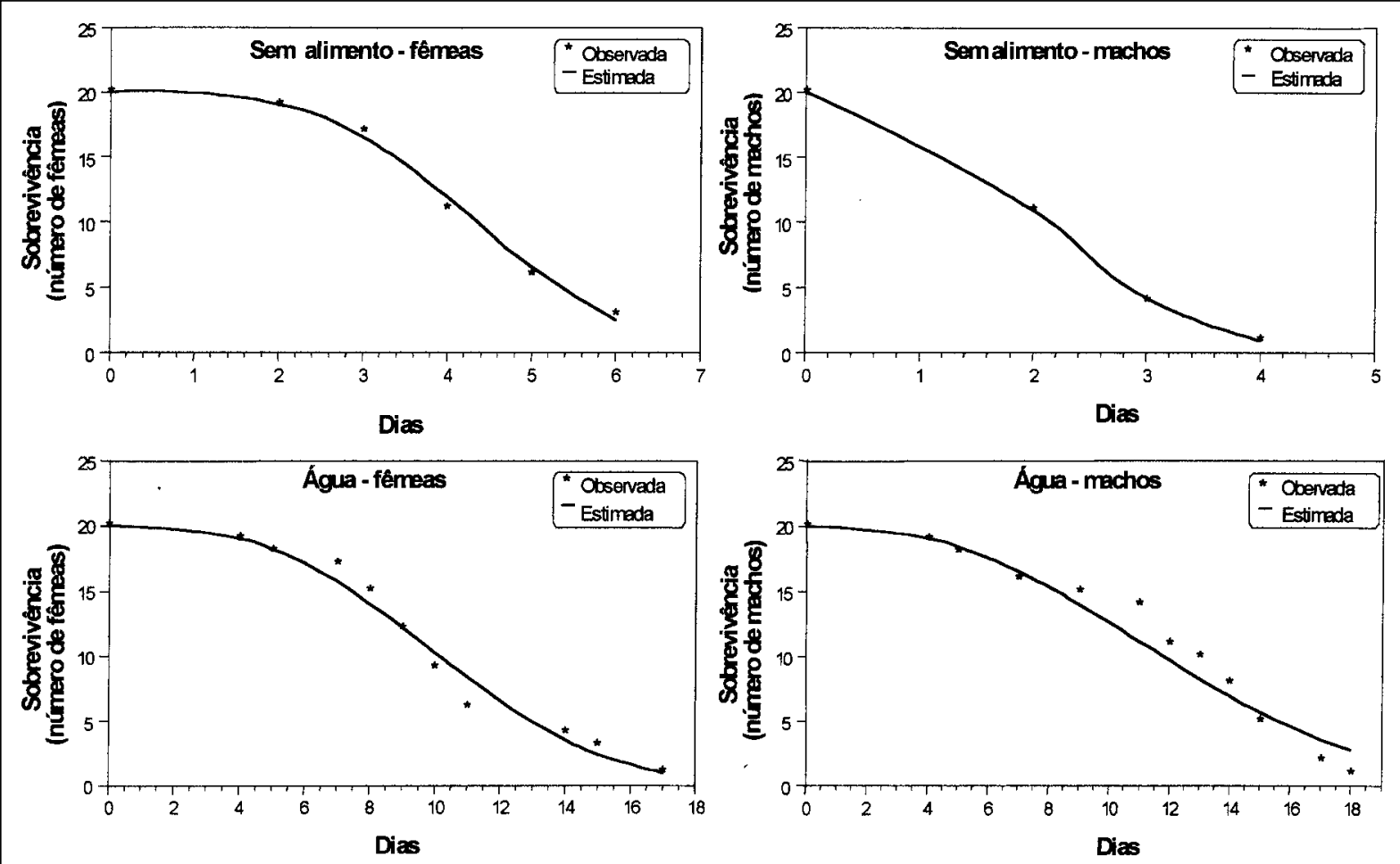

Figura 1 - Longevidade de fêmeas e machos de Bonagota cranaodes na ausência de alimento e em água. Temperatura de $25 \pm 1^{\circ} \mathrm{C}$ e $70 \pm 10 \%$ de UR e fotofase de 12 h, Laboratório de Biologia dos Insetos FAEM/UFPel.
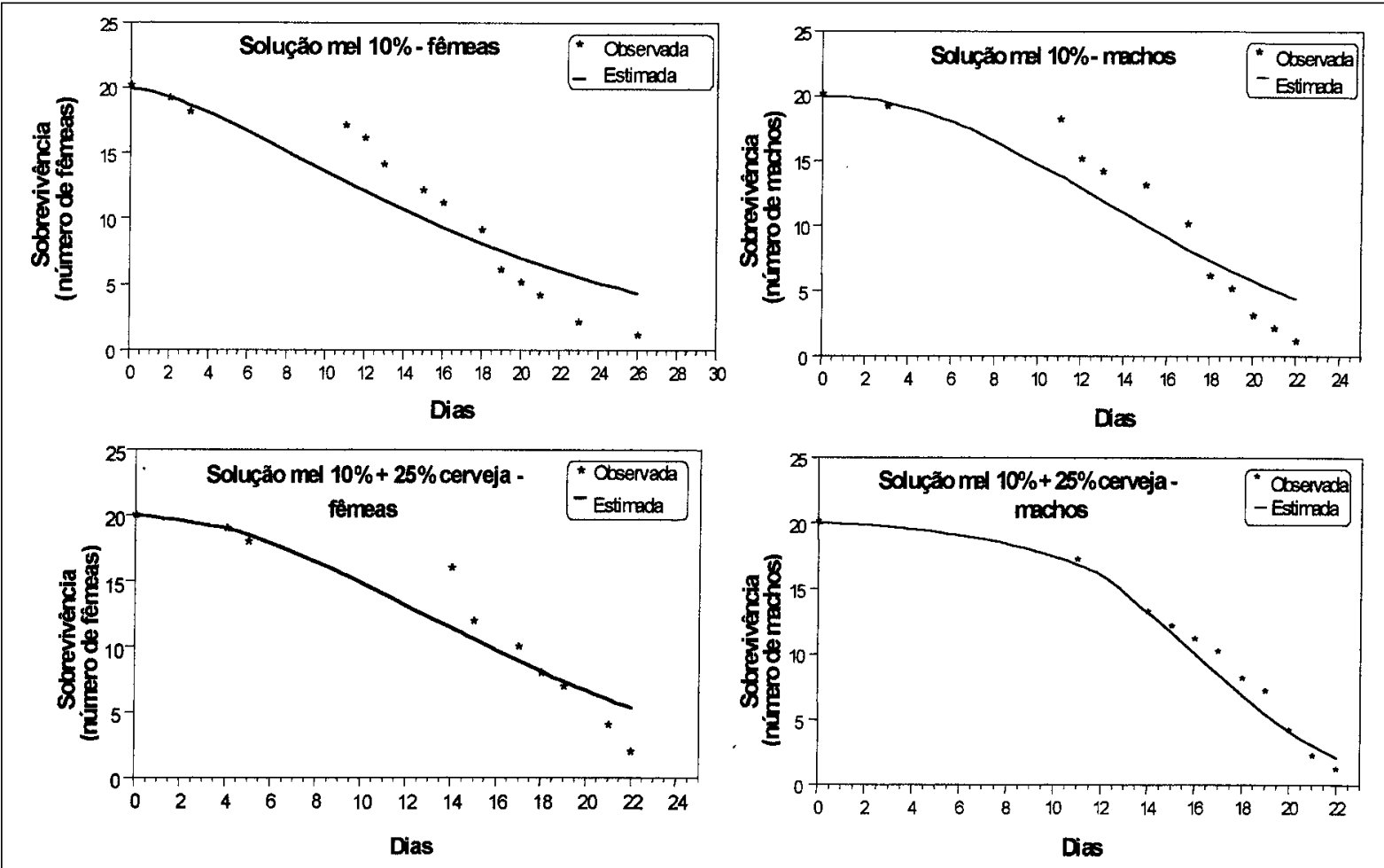

Figura 2 - Longevidade de fêmeas e machos de Bonagota cranaodes em solução de mel a $10 \%$ e solução de mel $10 \%+25 \%$ de cerveja. Temperatura de $25 \pm 1^{\circ} \mathrm{C}$ e $70 \pm 10 \%$ de UR e fotofase de $12 \mathrm{~h}$, Laboratório de Biologia dos Insetos FAEM/UFPel.

Ciência Rural, v.35, n.6, nov-dez, 2005. 
e a maior capacidade de postura (291 ovos/fêmea), sem reduzir significativamente a longevidade dos adultos em relação às demais dietas.

\section{CONCLUSÕES}

Os resultados mostram que as fêmeas não necessitam de água e/ou mel para iniciar atividade reprodutiva, sendo que a sobrevivência é reduzida na ausência desses recursos. As dietas segundo as quais os adultos foram alimentados com solução de mel a $10 \%$ e solução de mel a $10 \%$ + cerveja a $25 \%$ são as mais indicadas para a alimentação dos adultos de Bonagota cranaodes em laboratório. A dieta com solução de mel a $10 \%$ por ter um custo menor é a mais adequada para a manutenção dos adultos da lagarta enroladeira em laboratório.

\section{REFERÊNCIAS}

BAVARESCO, A. Efeito de dietas naturais e artificiais sobre o desenvolvimento de Spodoptera latifascia (Walker, 1856) (Lepidoptera: Noctuidae). 2000. $83 \mathrm{f}$. Dissertação (Mestrado em Fitossanidade) - Curso de Pósgraduação em Fitossanidade, Universidade Federal de Pelotas.

BENZ, G. Physiology reproduction and ecology. In: VANDER GEEST, L.P.S; EVENHUIS H.H. Pests tortricid pests their biology, natural enemies and control. Amsterdam: Elsevier Science, 1991. 808p.

EIRAS, A.E. et al. Biologia comparada da lagarta enroladeira Phtheochroa cranaodes (Meyrick) em duas dietas artificiais. Anais da Sociedade Entomológica do Brasil, São Paulo, v.23, n.2, p.251-257, 1994

FONSECA, F.L. et al. Influência da dieta na sobrevivência e fecundidade de adultos de Bonagota cranaodes (Meyrick) (Lepidoptera: Tortricidae) em condições de laboratório. In. CONGRESSO BRASILEIRO DE ENTOMOLOGIA, 17., 1998,
Rio de Janeiro, RJ. Anais... Rio de Janeiro: Universidade Federal Rural do Rio de Janeiro, 1998. p.942.

GARCIA, M.S. Bioecologia e potencial de controle biológico de Ecdytolopha aurantiana (Lima, 1927) (Lepidoptera: Tortricidae), o bicho-furão, através de Trichogramma pretiosum, 1879. 1998. 118f. Tese (Doutorado em Entomologia) - Curso de Pós-graduação em Entomologia, Escola Superior de Agricultura “Luiz de Queiroz”/USP.

PANIZZI, A.R.; PARRA, J.R.P. Ecologia nutricional de insetos e suas implicações no manejo de pragas. São Paulo: Manole, 1991. 359p.

PARRA, J.R. et al. Técnica de criação de Phtheochroa cranaodes Meyrick (Lepidoptera: Tortricidae) em dieta artificial. Revista Brasileira de Biologia. São Paulo, v.55, n.4, p.537-543, 1995.

PARRA, J.R.P. et al. Efeito da nutrição de adultos e da umidade na fecundidade de Diatraea saccharalis (Fabr.) (Lepidoptera: Crambidae). Anais da Sociedade Entomológica do Brasil, São Paulo, v.28, n.1, p.49-57, 1999

PARRA, J.R.P. A biologia de insetos e o manejo de pragas: da criação em laboratório a aplicação em campo. In: GUEDES, J.C. et al. Bases técnicas do manejo de insetos. Santa Maria: UFSM/CCR/DFS, Pallotti, 2000. 248p. p.1-29.

SAS. User's guide: statistics. 5.ed. Cary, NC: SAS Institute, 1985. 965p.

SCRIBER, J.M.; SLANSKY, Jr. F. The nutritional ecology of immature insects. Annual Review of Entomolology, Palo Alto, v.26, p.183-211, 1981

SGRILLO, R.B. A distribuição de Weibull como modelo de sobrevivência de insetos. Ecossistema, Espírito Santo do Pinhal, v.7, p.10-13, 1982.

SILVA, R.A. da et al. Efeitos de diferentes dietas na biologia da fase adulta de Mocis latipes (Guenée, 1852) (Lepidoptera: Noctuidae). Pesquisa Agropecuária Brasileira, Brasília, v.26, n.1, 1991. 